\title{
Too Cold to Clot? Does Intraoperative Hypothermia Contribute to Bleeding After Aortic Surgery?
}

\author{
Louis H. Stein, MD, PhD ${ }^{1}$, Gregory Rubinfeld, BA², Leora B. Balsam, MD²*, \\ Patricia Ursomanno, $\mathrm{PhD}^{3}$, Abe DeAnda, Jr., MD ${ }^{4}$ \\ 1 Division of Cardiothoracic Surgery, SUNY Downstate Medical Center, Brooklyn, New York, USA \\ ${ }^{2}$ Department of Cardiothoracic Surgery, NYU Langone Medical Center, New York, New York, USA \\ ${ }^{3}$ Maimonides Heart and Vascular Center, Maimonides Medical Center, Brooklyn, New York, USA \\ ${ }^{4}$ Division of Cardiothoracic Surgery, University of Texas Medical Branch-Galveston, Galveston, Texas, USA
}

\begin{abstract}
Background: We determined the impact of intraoperative hypothermia on postoperative bleeding after thoracic aortic surgery.

Methods: We retrospectively analyzed 98 consecutive patients who underwent aortic surgery with deep hypothermic circulatory arrest between 2010 and 2014. We evaluated lowest temperature, absolute decrease in temperature, and rewarming rate. Univariate and multivariate regression were used to determine relationships between temperature, clinical characteristics, and measures of postoperative bleeding.

Results: The mean age of patients was $60.5 \pm 15.1$ years, with $64.3 \%$ male and $60 \%$ Caucasian. The lowest temperatures recorded were $13.5 \pm 4.6^{\circ} \mathrm{C}$ at the bypass circuit. Change in hematocrit was associated with ethnicity, preoperative hematocrit, and rewarming rate. Chest tube output was associated with body mass index, preoperative platelet count, prior cardiac surgery, cardiopulmonary bypass (CPB) duration, intraoperative blood product transfusion, lowest surface temperature, and change in surface temperature. Postoperative packed red blood cell transfusion was associated with ejection fraction, chronic obstructive pulmonary disease (COPD), platelet count, partial thromboplastin time, CPB duration, and lowest blood temperature. Fresh frozen plasma transfusion correlated with COPD, CPB duration, and final blood temperature. Platelet transfusion correlated with body mass index and preoperative platelet count. Unplanned reoperation for
\end{abstract}

Published by Science International Corp. ISSN 2325-4637

Fax +12037853552

E-Mail: aorta@scienceinternational.org

http://aorta.scienceinternational.org

Accessible online at:

http://aorta.scienceinternational.org bleeding was associated with final temperature and change in temperature.

Conclusion: We found no consistent associations between intraoperative temperature and indicators of bleeding. Intraoperative cooling strategies should be based on optimal end-organ protection rather than fear of postoperative bleeding; rewarming strategies may ameliorate the risk of coagulopathy.

Copyright () 2017 Science International Corp.

\section{Key Words}

Aorta $\cdot$ Postoperative hemorrhage $\cdot$ Hypothermia

\section{Introduction}

Hypothermia is an effective method of brain and systemic end-organ protection during thoracic aortic surgery [1-4], most likely due to decreased metabolic requirements during periods of ischemia [4]. Although deeper levels of hypothermia allow for longer periods of safe circulatory arrest [3, 4], hypothermia is not without risks. In particular, coagulopathy related to hypothermia is a concern to the surgeon.

In vivo animal models [5] and clinical studies [6] demonstrated an association between coagulopathy and hypothermia. Laboratory studies show that decreased temperature inhibits platelet function $[7,8]$ and the activity of enzymes involved in the co-

* Corresponding Author:

Leora Balsam, MD

Department of Cardiothoracic Surgery

NYU Langone Medical Center

530 First Ave, Suite 9V New York, NY 10016, USA

Tel.: +1 212263 7835; Fax: +1 212263 2042; E-Mail: leora.balsam@nyumc.org 
agulation cascade $[1,9,10]$. Although these inhibitory effects may be incomplete due to intrinsic redundancy in the coagulation mechanism, bleeding and the need for transfusions are both associated with increased postoperative morbidity and mortality [11].

Aortic reconstructive surgery typically involves the use of cardiopulmonary bypass (CPB), long suture lines, release of tissue plasminogen activator in aorto-intimal disease, and in some cases, preoperative anticoagulation, all of which can predispose patients to postoperative bleeding [12-16]. Thus, the surgeon is faced with the dilemma of balancing the use of hypothermia for end-organ protection with the theoretical concern of worsening coagulopathy. Given these concerns, some surgeons curtail their use of hypothermic circulatory arrest to decrease the risk of postoperative bleeding.

The effect of intraoperative hypothermia on postoperative bleeding is not well characterized. Whereas coagulopathy is clearly present during episodes of hypothermia, it is unclear how readily coagulation returns to clinically normal function after rewarming. Here, we hypothesized that the depth of hypothermia does not affect postoperative bleeding as long as rewarming is achieved prior to separation from CPB.

\section{Materials and Methods}

\section{Patient Population}

Outcomes of 98 consecutive patients who underwent open surgical repair of dissections or aneurysms of the thoracic aorta using deep hypothermic circulatory arrest (DHCA) at our institution from January 2010 to January 2014 were retrospectively reviewed. Inclusion criteria were surgery of the root, ascending, transverse arch, or descending aorta and age $\geq 16$ years. Indications for surgery included clinical and radiologic evidence of rupture or dissection, aneurysm growth $\geq 0.5 \mathrm{~cm}$ in 6 months, symptomatic aneurysms, or aneurysms $\geq 5.5 \mathrm{~cm}$. Institutional review board approval for de-identified patient outcome data analysis was obtained, and the requirement for written informed consent was waived.

\section{Surgical Technique and Management \\ Intraoperative management of patients}

undergoing aortic surgery at our institution has previously been described $[17,18]$. Techniques for intraoperative blood conservation were utilized. All patients received an antifibrinolytic (Amicar, Clover Pharmaceuticals Corp. Marietta, GA) as part of their intraoperative management. Other adjuncts (e.g., desmopressin, recombinant factor VIla) were not used. Patients underwent a period of DHCA with variable target nadir temperatures based on the planned procedure and surgeon preference. In procedures involving the aortic arch, selective antegrade cerebral perfusion was used, although it did not alter the targeted nadir temperature. Rewarming was actively performed to achieve separation from cardiopulmonary bypass at a target core temperature of $>37^{\circ} \mathrm{C}$. The postoperative transfusion of blood products was performed in accordance with the Society of Thoracic Surgeons/Society of Cardiovascular Anesthesiologist 2011 guidelines [19]. We employed a low threshold to return to the operating room for bleeding.

\section{Demographic and Intraoperative Characteristics}

Baseline characteristics of each patient were collected. Data included age, gender, ethnicity, comorbid conditions, use of anticoagulant medications, preoperative international normalized ratio (INR), partial thromboplastin time (PTT), hematocrit, platelet count, and surgical priority (emergent, urgent, or elective). Intraoperative measurements included CPB duration, cross clamp (XC) duration, circulatory arrest duration, and patient temperature. Temperature was measured intraoperatively at the venous blood return to the CPB circuit. Tympanic membrane, bladder, rectal, or esophageal temperature was recorded as "body surface temperature" (Figure 1). The choice of body surface temperature recorded was left to the discretion of the perfusionist and/or anesthesiologist. For the present analysis, only one source of body surface temperature was recorded for each patient. Temperature variables recorded included lowest intraoperative temperature $\left(T_{\text {Low }}\right)$, temperature at the time of separation from bypass $\left(T_{\text {Fin }}\right)$, change in temperature $\left(T_{\text {Fin }}-T_{\text {Low }} ; \Delta T\right)$, and rewarming rate $(\Delta T /$ time from lowest temperature to separation from $C P B$ ) (Table 1). 


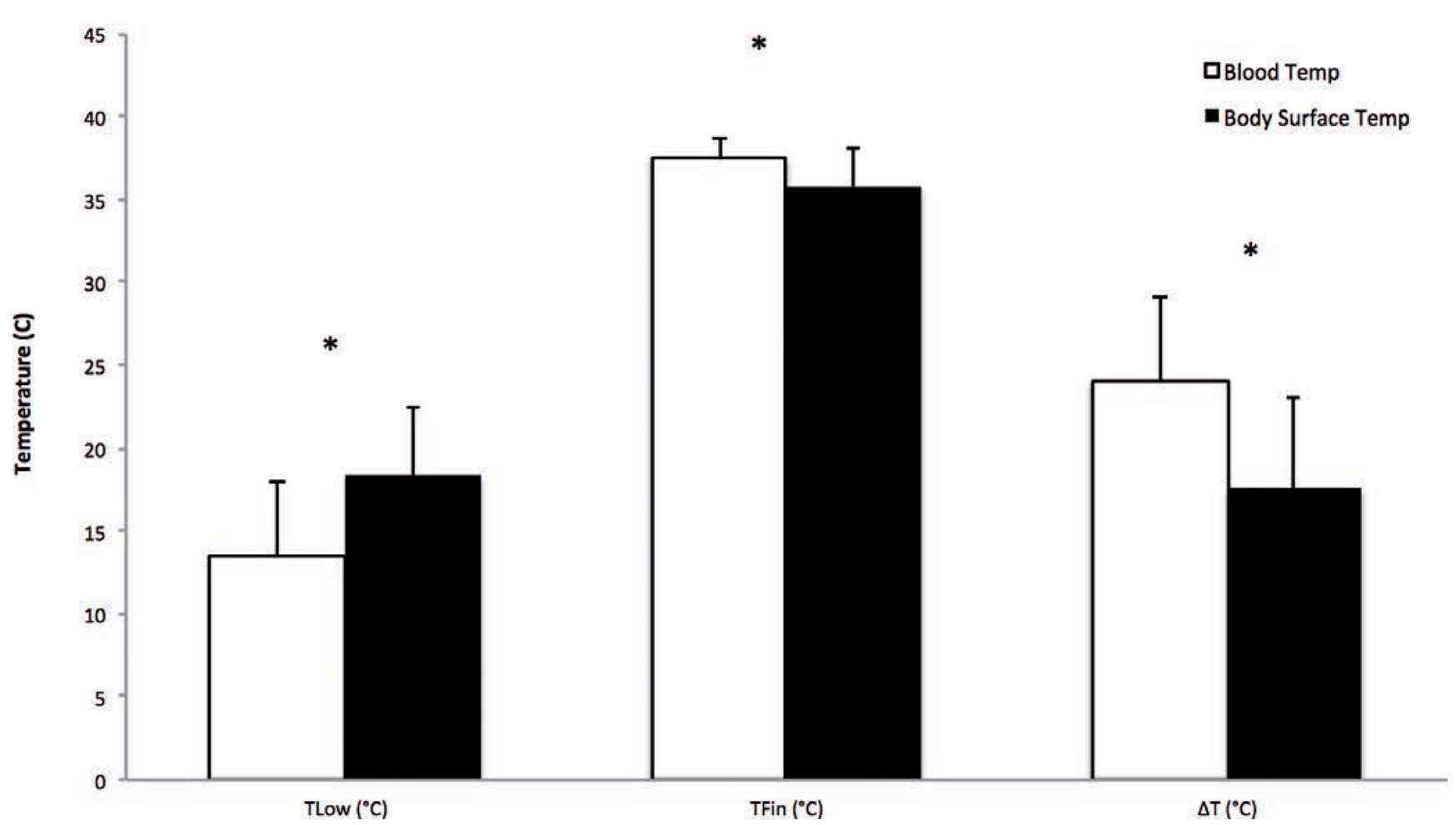

Figure 1. Significant difference between temperature measured from venous return blood and the patient's body surface.

\section{Measures of Postoperative Bleeding}

Outcomes measured included change in hematocrit at $1 \mathrm{~h}\left(\triangle \mathrm{HCT}_{1}\right)$ and $12 \mathrm{~h}\left(\triangle \mathrm{HCT}_{12}\right)$ postoperatively, chest tube (CT) output at $12 \mathrm{~h}$ postoperatively, postoperative transfusion requirements, and unplanned return to the operating room due to bleeding. We also constructed a cumulative measure of significant bleeding based on previously described criteria [20]. Significant bleeding was defined as $>800 \mathrm{cc}$ of CT drainage in $12 \mathrm{~h}$, transfusion of more than three units of packed red blood cells (PRBCs) or fresh frozen plasma (FFP), any transfusion of cryoprecipitate or platelets, or unplanned return to the operating room due to bleeding.

\section{Statistical Analysis}

Baseline and clinical data are presented as mean \pm standard deviation for continuous variables or percentage of the respective cohort for categorical variables. Because transfusion data were not normally distributed, they are expressed as mean, median, and range. Univariate and multivariate linear regression were used to determine correlations between clinical characteristics and measures of postoperative bleed- ing $(\triangle \mathrm{HCT})$. Logistic regression was performed to determine the impact of clinical variables on the odds of transfusion consistent with significant bleeding and reoperation for postoperative bleeding. CT output demonstrated a left-skewed distribution; therefore, data for volume output was $\log _{10}$-transformed to satisfy the parametric requirements for regression analysis. Because of the zero-inflated distribution of postoperative transfusion, correlations between blood product transfusion and clinical variables were performed using generalized linear modeling with a negative binomial distribution. Univariate regression was used to determine the correlation between individual covariates and outcomes. Correlations between covariates and the odds of significant bleeding were performed using logistic regression analysis. Regression analysis was first performed using a univariate model. Covariates showing a significant correlation $(p<0.05)$ were included in multivariate models in a forward stepwise fashion. Covariates with collinearity tolerance $<0.1$ were further evaluated as part of the construction of multivariate models. Statistical analysis was conducted using the Statistical Package for the Social Sciences (SPSS, Version 20, IBM, Armonk, New York). 


\section{Results}

\section{Demographic and Intraoperative Characteristics}

Ninety-eight patients who underwent thoracic aortic surgery with DHCA during the study period met inclusion criteria. The mean age of patients was $60.5 \pm 15.1$ years, and $64.3 \%$ were male (Table 3 ). Significant comorbidities included diabetes in $21 \%$ and chronic obstructive pulmonary disease (COPD) in $44 \%$ of patients. Left ventricular ejection fraction (EF) was $54 \pm 15 \%$, and mean systolic blood pressure was $129 \pm 20 \mathrm{mmHg}$. A third of the patients were taking aspirin preoperatively, and $<10 \%$ were taking another anticoagulant or antiplatelet medication. Mean platelet count, hematocrit, INR, and PTT were in the normal range. Prior cardiac surgery was noted in $18 \%$ of patients. Most patients had aneurysmal disease rather than dissection ( $84 \%$ vs. $16 \%$ ). Among patients undergoing repair for aneurysmal disease, $46 \%$

Table 1. Temperatures measured at the venous return and body surface differ significantly.

\begin{tabular}{llll}
\hline Variable & Blood & Body Surface & p-value \\
\hline$T_{\text {Low }}\left({ }^{\circ} \mathrm{C}\right)$ & $13.47 \pm 0.47$ & $18.28 \pm 0.44$ & $<0.001$ \\
$T_{\text {Fin }}\left({ }^{\circ} \mathrm{C}\right)$ & $37.47 \pm 1.30$ & $35.77 \pm 2.34$ & $<0.001$ \\
$\Delta \mathrm{T}\left({ }^{\circ} \mathrm{C}\right)$ & $23.95 \pm 0.54$ & $17.53 \pm 0.57$ & $<0.001$ \\
Rewarm rate & $0.29 \pm 0.16$ & $0.21 \pm 0.09$ & $<0.001$ \\
$\left({ }^{\circ} \mathrm{C} \times \mathrm{min}^{-1}\right)$ & & & \\
\hline
\end{tabular}

Table 2. Intraoperative characteristics of patients included in this study.

\begin{tabular}{lc}
\hline Variable & Total Cases $\mathbf{n}=\mathbf{9 8}$ \\
\hline Valve replacement & $31.6 \%(31)$ \\
Proximal & $82.7 \%(81)$ \\
Total Arch & $2 \%(2)$ \\
Descending & $13.2 \%(13)$ \\
Thoracoabdominal & $10.2 \%(10)$ \\
CPB (min) & $175.3 \pm 58.9$ \\
xC (min) & $85.5 \pm 61.3$ \\
Circulatory arrest (min) & $23.5 \pm 14.9$ \\
\hline Continuous data are shown as mean \pm standard deviation. Categorical data \\
are shown as percent (number). \\
CPB = cardiopulmonary bypass time; xc= cross clamp time. \\
\hline
\end{tabular}

Stein, L. H. et al. were elective, $28 \%$ were urgent, and $26 \%$ were emergent. Six patients were hemodynamically unstable at the time of presentation. Compared with patients with aneurysms, a significantly larger proportion of patients with dissections presented with hemodynamic instability ( $4 \%$ vs. $18 \% ; p=0.02$ ). Most dissection patients underwent emergent repair (69\%), whereas fewer underwent urgent $(25 \%)$ or elective $(6 \%)$ procedures.

Most patients required replacement of the proximal aorta (Table 2). CPB duration was $175.3 \pm 58.9 \mathrm{~min}$, and myocardial ischemic duration was $85.5 \pm 61.3$

Table 3. Baseline characteristics of patients included in the present study.

\begin{tabular}{|c|c|}
\hline Variable & Total Cases $n=98$ \\
\hline Age (years) & $60.5 \pm 15.1$ \\
\hline Male (\%) & $64.3 \%(63)$ \\
\hline \multicolumn{2}{|l|}{ Race (\%) } \\
\hline White & $60.2 \%(59)$ \\
\hline Black & $32.7 \%(32)$ \\
\hline Asian & $5.1 \%(5)$ \\
\hline Diabetes & $21.4 \%(21)$ \\
\hline COPD & $43.8 \%(43)$ \\
\hline BMI & $27.4 \pm 5.0$ \\
\hline LVEF & $54.5 \pm 15.5$ \\
\hline $\mathrm{BP}$ & $129 / 69 \pm 20 / 13$ \\
\hline \multicolumn{2}{|c|}{ Preop anticoagulation } \\
\hline ASA & $33.71 \%$ (33) \\
\hline Plavix & $8.2 \%(8)$ \\
\hline Coumadin & $8.2 \%(8)$ \\
\hline Creatinine & $1.75 \pm 0.83$ \\
\hline INR (preop) & $0.9 \pm 0.4$ \\
\hline PTT (preop) & $33.1 \pm 12.1$ \\
\hline HCT (preop) & $35.9 \pm 6.2$ \\
\hline Plt (preop) & $199.0 \pm 69.0$ \\
\hline Reoperation & $18.3 \%(18)$ \\
\hline Dissection & $16.3 \%(16)$ \\
\hline \multicolumn{2}{|c|}{$\begin{array}{l}\text { Continuous data are shown as mean } \pm \text { standard deviation. Categorical data } \\
\text { are shown as percent (number). } \\
\text { COPD = chronic obstructive pulmonary disease; BMI = body mass index; } \\
\text { LVEF = left ventricular ejection fraction; BP = blood pressure; ASA = atrial } \\
\text { septal aneurysm; INR = international normalized ratio; PTT = partial throm- } \\
\text { boplastin time; HCT = hematocrit; Plt = platelet. }\end{array}$} \\
\hline
\end{tabular}


min. Total circulatory arrest duration, which included periods of selective antegrade cerebral perfusion, was $23.5 \pm 14.9 \mathrm{~min}$.

\section{Effects of Baseline and Operative Characteristics on Postoperative Bleeding}

Hematocrit. Hematocrit decreased significantly from $36.3 \pm 6 \%$ preoperatively, to $31.4 \pm 6 \% 1 \mathrm{~h}$ postoperatively, to $27.2 \pm 5 \% 12 \mathrm{~h}$ postoperatively $(\mathrm{p}<0.05) . \Delta \mathrm{HCT}_{12}$ was larger than $\triangle \mathrm{HCT}_{1}(8.9 \pm 6.4$ vs. $4.6 \pm 6.1$, respectively; $\mathrm{p}<0.001)$. $\Delta \mathrm{HCT}_{1}$ was significantly associated with female gender, non-white ethnicity, preoperative hematocrit, and preoperative platelet count in univariate linear regression (Table 4) but was associated only with preoperative hematocrit in multivariate linear regression (coefficient: 0.59 , $\mathrm{p}<0.01$ ). $\triangle \mathrm{HCT}_{12}$ was significantly associated with female gender, non-white ethnicity, presence of dissection, preoperative hematocrit, intraoperative transfusion of PRBCs, and blood temperature rewarming rate in univariate linear regression (Table 4) and was associated with female gender (coefficient: $-2.35, \mathrm{p}=$ 0.014 ), presence of dissection (coefficient: $2.77, p=$ 0.02 ), and preoperative hematocrit (coefficient: 0.71 , $p<0.001)$ in multivariate linear regression

CT Drainage. CT output is a universally utilized measure for assessing ongoing postoperative bleeding. We examined total CT output at $12 \mathrm{~h}$, as we felt this time period was most reflective of postoperative bleeding and coagulopathy. Mean CT output was $831 \pm 724 \mathrm{ml}$ (median: $595.0 \mathrm{ml}$; range 50-3,830 ml). Univariate linear regression showed that proximal and descending aortic disease, $\mathrm{BMI}$, preoperative platelet count, CPB duration, reoperation, and intraoperative transfusion of PRBCs, platelets, or FFP were significantly associated with $\log _{10}$-transformed CT output (Table 5). Multiple linear regression showed that CPB duration (coefficient: $0.003 \pm 0.001, p=0.001$ ), preoperative platelet count (coefficient: $-0.002 \pm 0.001, p=0.001$ ), descending disease (coefficient: $0.271 \pm 0.104, p=0.012$ ), and intraoperative PRBC transfusion (coefficient: $0.029 \pm$ $0.013, p=0.036$ ) were associated with CT output.

Transfusion Requirements. Transfusion requirements for 7 days following the index surgery are summarized in Supplementary Table 1. The data were rightskewed with notable zero-inflated data; therefore, we used generalized linear modeling with a negative bi- nomial distribution to determine the clinical factors associated with intraoperative and postoperative transfusion (Table 6). Postoperative PRBC transfusion was significantly associated with EF, preoperative PTT, hematocrit, COPD, CPB duration, and lowest blood temperature. In multivariate analysis, only $\mathrm{CPB}$ duration (coefficient: $0.017, p<0.001)$ and PTT $(0.053 ; p=0.003)$ were significantly associated with postoperative PRBC transfusion. No temperature measurements were significant predictors of FFP or platelet transfusion.

Reoperation for Bleeding. Twelve patients (12.2\%) required reoperation for postoperative bleeding. Univariate logistic regression showed that change in blood temperature and final blood temperature were significantly associated with the odds of unplanned re-operation for bleeding (Table 6).

Significant Postoperative Bleeding. Fifty-one patients met our cumulative criteria for significant postoperative bleeding. Univariate logistic regression showed six factors that were significantly associated with the odds of postoperative bleeding: preoperative use of any form of anticoagulation, pre-operative platelet count, CPB time, and transfusion of PRBCs, platelets, or FFP (Supplementary Table 2). No measurement of temperature was associated with significant postoperative bleeding. Multivariate logistic regression showed that CPB duration (coefficient: 0.02 , odds ratio (OR): $1.012(1.002-1.022), p=$ 0.02 ) and pre-operative platelet count (coefficient: -0.02 , OR $0.99(0.98-0.99), p=0.006)$ were significantly associated with postoperative bleeding.

\section{Discussion}

The use of hypothermic circulatory arrest for end-organ protection is tempered by concerns for postoperative coagulopathy and resultant bleeding. Clinicians may "know" what bleeding is, but a collectively agreed upon definition remains elusive. Therefore, we evaluated postoperative blood loss from two different viewpoints: postoperative parameters indicative of bleeding and postoperative treatments used to address ongoing blood loss.

We focused on four measures that are reflective of postoperative bleeding: change in hematocrit, CT output, transfusion requirement, and re-operation for bleeding. Hematocrit is a nearly universally utilized clinical measure of circulating blood volume. 
Table 4. Correlation between clinical characteristics and temperature measures with change in hematocrit at $1 \mathrm{~h}$ and $12 \mathrm{~h}$ postoperatively. Univariate linear regression presented as regression coefficient \pm standard deviation with associated $p$-value.

\begin{tabular}{|c|c|c|c|c|}
\hline \multirow[b]{2}{*}{ Covariate } & \multicolumn{2}{|c|}{$\Delta \mathrm{HCT} 1 \mathrm{~h}$} & \multicolumn{2}{|c|}{$\Delta \mathrm{HCT} 12 \mathrm{~h}$} \\
\hline & Coefficient \pm SD & p-value & Coefficient \pm SD & p-value \\
\hline Age & $-0.02 \pm 0.04$ & 0.59 & $-0.08 \pm 0.04$ & 0.06 \\
\hline Gender & $-3.0 \pm 1.28$ & 0.02 & $-4.4 \pm 1.32$ & 0.001 \\
\hline Non white & $-2.63 \pm 1.24$ & 0.04 & $-2.98 \pm 1.32$ & 0.03 \\
\hline Any anticoagulation & $1.30 \pm 1.24$ & 0.30 & $0.48 \pm 1.35$ & 0.73 \\
\hline Dissection & $-0.36 \pm 1.68$ & 0.83 & $3.56 \pm 1.78$ & 0.05 \\
\hline Valve & $-0.69 \pm 1.35$ & 0.61 & $-1.28 \pm 1.44$ & 0.37 \\
\hline Proximal & $0.38 \pm 1.64$ & 0.82 & $-0.45 \pm 1.87$ & 0.81 \\
\hline Descending & $0.61 \pm 1.83$ & 0.74 & $2.11 \pm 2.06$ & 0.31 \\
\hline Thoracoabdominal & $0.59 \pm 1.79$ & 0.74 & $-0.39 \pm 1.88$ & 0.84 \\
\hline Priority & $-0.24 \pm 0.74$ & 0.75 & $0.73 \pm 0.77$ & 0.35 \\
\hline $\mathrm{BMI}$ & $-0.19 \pm 0.12$ & 0.14 & $0.03 \pm 0.14$ & 0.85 \\
\hline $\mathrm{EF}$ & $0.07 \pm 0.04$ & 0.07 & $0.05 \pm 0.04$ & 0.29 \\
\hline Creatinine & $-0.05 \pm 0.46$ & 0.91 & $-0.13 \pm 0.49$ & 0.79 \\
\hline HCT (preop) & $0.58 \pm 0.08$ & 0.00 & $0.75 \pm 0.08$ & 0.00 \\
\hline INR (preop) & $1.67 \pm 1.67$ & 0.32 & $2.37 \pm 1.89$ & 0.21 \\
\hline PTT (preop) & $0.09 \pm 0.06$ & 0.09 & $-0.01 \pm 0.06$ & 0.87 \\
\hline Plt (preop) & $-0.02 \pm 0.01$ & 0.03 & $-0.02 \pm 0.01$ & 0.09 \\
\hline COPD & $-1.75 \pm 1.21$ & 0.15 & $-0.35 \pm 1.27$ & 0.78 \\
\hline Diabetes & $-1.78 \pm 2.25$ & 0.43 & $-0.003 \pm 2.3$ & 0.99 \\
\hline Dialysis & $-2.02 \pm 3.37$ & 0.55 & $-2.24 \pm 3.23$ & 0.49 \\
\hline CPB & $0.00 \pm 0.01$ & 0.99 & $-0.012 \pm 0.011$ & 0.28 \\
\hline Cross clamp & $0.04 \pm 0.01$ & 0.67 & $-0.004 \pm 0.011$ & 0.66 \\
\hline Circulatory Arrest & $-0.03 \pm 0.04$ & 0.46 & $0.001 \pm 0.003$ & 0.98 \\
\hline PRBC (intraop) & $-0.31 \pm 0.20$ & 0.12 & $-0.52 \pm 0.21$ & 0.01 \\
\hline PRBC (postop) & $0.25 \pm 0.24$ & 0.29 & $0.012 \pm 0.04$ & 0.83 \\
\hline FFP (intraop) & $0.72 \pm 0.46$ & 0.12 & $0.25 \pm 0.52$ & 0.62 \\
\hline Plt (intraop) & $1.03 \pm 0.06$ & 0.09 & $0.54 \pm 0.66$ & 0.42 \\
\hline$T_{\text {Low }}($ blood $)$ & $0.10 \pm 0.14$ & 0.46 & $-0.072 \pm 0.16$ & 0.65 \\
\hline $\mathrm{T}_{\text {Fin }}$ (blood) & $-0.76 \pm 0.48$ & 0.11 & $-0.48 \pm 0.51$ & 0.36 \\
\hline$\Delta \mathrm{T}$ (blood) & $-0.11 \pm 0.12$ & 0.36 & $0.04 \pm 0.14$ & 0.79 \\
\hline Rewarming rate (blood) & $6.96 \pm 3.89$ & 0.07 & $10.89 \pm 4.12$ & 0.01 \\
\hline$T_{\text {Low }}$ (surface) & $-0.07 \pm 0.15$ & 0.62 & $-0.018 \pm 0.16$ & 0.91 \\
\hline $\mathrm{T}_{\text {Fin }}$ (surface) & $0.32 \pm 0.28$ & 0.24 & $0.093 \pm 0.29$ & 0.75 \\
\hline$\Delta T$ (surface) & $0.09 \pm 0.11$ & 0.46 & $0.018 \pm 0.12$ & 0.88 \\
\hline Rewarming rate (surface) & $6.14 \pm 6.39$ & 0.34 & $7.34 \pm 6.85$ & 0.29 \\
\hline
\end{tabular}

P-values of regression coefficient achieving statistical significance are in bold.

$\mathrm{BMI}=$ body mass index; $\mathrm{EF}=$ ejection fraction; $\mathrm{HCT}=$ hematocrit; $I \mathrm{NR}=$ international normalized ratio; PTT $=$ partial thromboplastin time; Plt $=$ platelet; $\mathrm{COPD}=$ chronic obstructive pulmonary disease; $\mathrm{LVEF}=$ left ventricular ejection fraction; $\mathrm{BP}=$ blood pressure; $\mathrm{ASA}=$ atrial septal aneurysm. 
Table 5. Univariate linear regression analysis of chest tube output.

\begin{tabular}{|c|c|c|}
\hline Variable & $\begin{array}{l}\text { Regression } \\
\text { Coefficient }\end{array}$ & p-value \\
\hline Age & -0.001 & 0.58 \\
\hline Gender & 013 & 0.112 \\
\hline Non white & -0.06 & 0.48 \\
\hline African American & -0.02 & 0.76 \\
\hline Surgeon & 0.01 & 0.54 \\
\hline Any anticoagulation & 0.14 & 0.68 \\
\hline Dissection & -0.02 & 0.87 \\
\hline Aneurysm & 0.02 & 0.87 \\
\hline Valve & -0.003 & 0.98 \\
\hline Proximal & -0.19 & 0.04 \\
\hline Descending & 0.25 & 0.02 \\
\hline Thoracoabdominal & 0.10 & 0.33 \\
\hline Priority & 0.05 & 0.90 \\
\hline BMI & -0.03 & 0.001 \\
\hline LVEF & 0.003 & 0.26 \\
\hline Creatinine & 0.034 & 0.28 \\
\hline INR (preop) & -0.06 & 0.54 \\
\hline PT (preop) & 0.015 & 0.52 \\
\hline PTT (preop) & 0.001 & 0.75 \\
\hline Plt (preop) & -0.001 & 0.05 \\
\hline COPD & 0.01 & 0.89 \\
\hline Diabetes & -0.15 & 0.21 \\
\hline Dialysis & 0.28 & 0.06 \\
\hline Reoperation & 0.02 & 0.04 \\
\hline CPB & 0.002 & 0.001 \\
\hline Cross clamp & 0.001 & 0.90 \\
\hline Circulatory Arrest & 0.003 & 0.28 \\
\hline RBC (intraop) & 0.38 & 0.002 \\
\hline Plt (intraop) & 0.07 & 0.05 \\
\hline FFP (intraop) & 0.06 & 0.042 \\
\hline $\mathrm{T}_{\text {Low }}(\mathrm{blood})$ & -0.003 & 0.68 \\
\hline $\mathrm{T}_{\text {Fin }}($ blood $)$ & -0.038 & 0.19 \\
\hline$\Delta \mathrm{T}$ (blood) & 0.001 & 0.91 \\
\hline Rewarming rate (Blood) & -0.35 & 0.13 \\
\hline $\mathrm{T}_{\text {Low }}$ (surface) & -0.02 & 0.014 \\
\hline$T_{\text {Fin }}$ (surface) & 0.008 & 0.63 \\
\hline$\Delta \mathrm{T}$ (surface) & 0.014 & 0.035 \\
\hline Rewarming rate (surface) & -0.32 & 0.41 \\
\hline
\end{tabular}

P-values of regression coefficient achieving statistical significance are in bold. $\mathrm{BMI}=$ body mass index; $\mathrm{LVEF}=$ left ventricular ejection fraction; INR = international normalized ratio; $\mathrm{PT}=$ prothrombin time; $\mathrm{PTT}=$ partial thromboplastin time; Plt = platelet; $\mathrm{COPD}=$ chronic obstructive pulmonary disease; $\mathrm{CPB}=$ cardiopulmonary bypass time; $\mathrm{RBC}=$ red blood cell; FFP = fresh frozen plasma.
Low hematocrit levels are associated with blood loss and may be triggers for blood transfusion. Because postoperative hematocrit is partly dependent on its preoperative level, we examined changes between pre- and postoperative levels. Given that hematocrit is affected by factors such as blood loss and dilution, we evaluated two different postoperative time points within the first $24 \mathrm{~h}$ after surgery. Factors previously associated with postoperative blood loss correlated with $\triangle \mathrm{HCT}$, but the only measure of temperature associated with increased $\triangle \mathrm{HCT}_{12}$ was a higher rewarming rate measured by blood temperature. This suggests caution in allowing a rate of rewarming above the generally recommended $0.2^{\circ} \mathrm{C} / \mathrm{min}$, although the mechanism for the association is unclear [21].

CT output is another commonly used measure of postoperative bleeding. Important caveats are that multiple factors can impact the volume of drainage, including tube position and non-blood fluid drainage. We found that lowest body surface temperature was negatively correlated with $\mathrm{CT}$ output, whereas the absolute change in body surface temperature was positively correlated with CT output. No single measure of blood temperature correlated with CT output. This may in part be due to lower peripheral temperatures relative to the temperature of circulating blood. In such a scenario, peripheral isolated compartments such as the pleura would remain cooler, and coagulopathy in these regions may persist.

Postoperative transfusion is reflective of a clinical concern for bleeding and coagulopathy. PRBC transfusion was related to lowest temperature of the blood, but this was not true for transfusions of platelets, FFP, or cryoprecipitate. This may be due to a clinical bias to have a lower threshold to transfuse PRBC in patients who were kept cooler due to concerns about hypothermia-related coagulopathy, even before clinical evidence of bleeding was apparent. The need to return to the operating room for bleeding reflects surgical bleeding and/or coagulopathy. Here, both final intraoperative blood temperature and change in temperature correlated with increased odds of return to the operating room due to bleeding. These factors did not remain significant when included in multivariate logistic regression.

We hypothesized that, with rewarming, there would be no correlation between the depth of hypo- 
Table 5. Generalized linear regression with negative binomial distribution analysis of postoperative transfusion.

\begin{tabular}{|c|c|c|c|c|c|c|}
\hline & \multicolumn{2}{|c|}{ PRBC } & \multicolumn{2}{|c|}{ FFP } & \multicolumn{2}{|c|}{ Plt } \\
\hline & Coeff & $p$-value & Coeff & $p$-value & Coeff & p-value \\
\hline Age & -0.09 & 0.67 & -0.004 & 0.69 & -0.11 & 0.91 \\
\hline Gender & -0.23 & 0.65 & -0.28 & 0.37 & -29.81 & 0.35 \\
\hline Non white & 0.13 & 0.81 & -0.08 & 0.81 & 10.34 & 0.72 \\
\hline Surgeon & 0.001 & 0.34 & 0.001 & 0.96 & 0.001 & 0.26 \\
\hline Any anticoagulation & 1.05 & 0.07 & 0.35 & 0.29 & -1.37 & 0.96 \\
\hline Dissection & -1.62 & 0.22 & -0.16 & 0.68 & -12.216 & 0.78 \\
\hline Valve & -0.176 & 0.25 & -0.57 & 0.15 & -20.67 & 0.53 \\
\hline Proximal & -0.227 & 0.18 & 0.123 & 0.78 & -27.4 & 0.43 \\
\hline Descending & 0.18 & 0.68 & -0.20 & 0.69 & -29.41 & 0.44 \\
\hline Thoracoabdominal & 0.42 & 0.51 & 0.42 & 0.51 & -23.77 & 0.49 \\
\hline Priority & -0.11 & 0.56 & -0.11 & 0.56 & -15.42 & 0.36 \\
\hline $\mathrm{BMI}$ & -0.06 & 0.95 & -0.06 & 0.95 & 0.014 & 0.00 \\
\hline $\mathrm{EF}$ & 0.013 & 0.05 & 0.013 & 0.05 & 0.405 & 0.59 \\
\hline Creatinine & -0.21 & 0.15 & -0.21 & 0.15 & 3.662 & 0.75 \\
\hline INR (preop) & -0.84 & 0.89 & -0.84 & 0.89 & -2.36 & 0.93 \\
\hline PTT (preop) & 0.08 & 0.05 & 0.016 & 0.34 & -0.082 & 0.95 \\
\hline Plt (preop) & 0.002 & 0.68 & 0.002 & 0.68 & 0.493 & 0.05 \\
\hline HCT (preop) & -0.09 & 0.02 & -0.09 & 0.02 & -0.68 & 0.08 \\
\hline COPD & 0.02 & $<0.01$ & 0.02 & $<0.01$ & -18.4 & 0.65 \\
\hline Diabetes & 1.09 & 0.27 & 1.09 & 0.27 & -7.81 & .0 .88 \\
\hline Dialysis & -0.73 & 0.60 & -0.73 & 0.60 & -7.14 & 0.94 \\
\hline Reoperation & 1.17 & 0.18 & 0.53 & 0.18 & 29.42 & 0.43 \\
\hline CPB & 0.023 & $<0.01$ & 0.012 & $<0.01$ & 0.11 & 0.66 \\
\hline Cross clamp & 0.03 & 0.08 & 0.03 & 0.07 & 0.176 & 0.48 \\
\hline Circulatory Arrest & 0.009 & 0.59 & 0.002 & 0.78 & 0.35 & 0.73 \\
\hline $\mathrm{T}_{\text {Low }}($ blood $)$ & -0.1 & 0.02 & 0.012 & 0.62 & 3.26 & 0.34 \\
\hline $\mathrm{T}_{\text {Fin }}($ blood $)$ & 0.08 & 0.55 & -0.17 & 0.05 & -10.16 & 0.42 \\
\hline$\Delta \mathrm{T}$ (blood) & -0.01 & 0.28 & 0.022 & 0.30 & -2.89 & 0.34 \\
\hline Rewarming rate (blood) & -0.64 & 0.55 & -1.45 & 0.06 & -54.53 & 0.42 \\
\hline $\mathrm{T}_{\text {Low }}($ surface $)$ & -0.03 & 0.17 & 0.031 & 0.38 & 0.12 & 0.97 \\
\hline $\mathrm{T}_{\text {Fin }}$ (surface) & 0.056 & 0.22 & 0.12 & 0.18 & -3.32 & 0.63 \\
\hline$\Delta \mathrm{T}$ (surface) & 0.031 & 0.08 & 0.031 & 0.23 & -0.646 & 0.82 \\
\hline Rewarming rate (surface) & -1.83 & 0.08 & -2.80 & 0.11 & -55.68 & 0.69 \\
\hline
\end{tabular}

P-values of regression coefficient achieving statistical significance are in bold.

$\mathrm{BMI}=$ body mass index; $\mathrm{EF}=$ ejection fraction; INR = international normalized ratio; PTT = partial thromboplastin time; Plt $=$ platelet; $\mathrm{HCT}=$ hematocrit; $\mathrm{COPD}=$ chronic obstructive pulmonary disease; $\mathrm{CPB}=$ cardiopulmonary bypass time. 
Table 6. Logistic regression requirement for reoperation for bleeding and clinical characteristics. Significant data presented as: regression coefficient; odds ratio ( $95 \%$ confidence interval).

\begin{tabular}{|c|c|c|c|}
\hline & Regression Coefficient & Odds Ratio (95\% CI) & $p$ value \\
\hline Age & -0.03 & $0.97(0.87-1.08)$ & 0.64 \\
\hline Gender & 0.85 & $2.33(1.07-50.98)$ & 0.59 \\
\hline Non white & -0.84 & $0.43(0.02-9.36)$ & 0.59 \\
\hline Any anticoagulation & 0.01 & $1.00(0.05-20.83)$ & 0.99 \\
\hline Valve & 0.84 & $2.33(0.11-50.98)$ & 0.59 \\
\hline Proximal & -0.41 & $0.67(0.03-14.03)$ & 0.79 \\
\hline Descending & 0.93 & $2.53(0.58-10.9)$ & 0.21 \\
\hline Thoracoabdominal & 1.94 & $6.96(0.54-89.06)$ & 0.14 \\
\hline Priority & -0.31 & $0.73(0.11-4.83)$ & 0.74 \\
\hline $\mathrm{BMI}$ & 0.09 & 1.09 (0.82-1.460 & 0.55 \\
\hline $\mathrm{EF}$ & -0.06 & $0.94(0.86-1.03)$ & 0.18 \\
\hline Creatinine & -0.91 & $0.40(0.02-9.05)$ & 0.57 \\
\hline HCT (preop) & -0.12 & $0.45(0.65-1.21)$ & 0.45 \\
\hline INR (preop) & 0.22 & $1.24(0.00-9.17)$ & 0.97 \\
\hline PTT (preop) & -0.09 & $0.91(0.68-1.22)$ & 0.53 \\
\hline Plt (preop) & 0.02 & $1.02(0.98-1.06)$ & 0.25 \\
\hline COPD & 1.24 & $3.46(1.09-10.88)$ & 0.03 \\
\hline Prior CT surgery & 0.56 & $1.57(0.38-6.59)$ & 0.53 \\
\hline $\mathrm{CPB}$ & 0.04 & $1.02(0.98-1.05)$ & 0.39 \\
\hline$T_{\text {Low }}($ blood $)$ & -0.15 & $0.86(0.72-1.01)$ & 0.07 \\
\hline $\mathrm{T}_{\text {Fin }}($ blood $)$ & 0.98 & $2.67(1.19-5.93)$ & 0.02 \\
\hline$\Delta \mathrm{T}$ (blood) & 0.17 & $1.18(1.18-1.39)$ & 0.04 \\
\hline Rewarming rate (blood) & 1.70 & $5.48(0.19-15.79)$ & 0.32 \\
\hline $\mathrm{T}_{\text {Low }}($ surface) & -7.73 & $0.00\left(0.00-3.4 \times 10^{3}\right)$ & 0.40 \\
\hline$T_{\text {Fin }}$ (surface) & 0.08 & $1.08(0.65-1.80)$ & 0.76 \\
\hline$\Delta \mathrm{T}$ (surface) & 0.18 & $1.19(0.45-3.19)$ & 0.72 \\
\hline Rewarming rate (surface) & -0.02 & $0.98(0.67-1.41)$ & 0.90 \\
\hline
\end{tabular}

P-values of regression coefficient achieving statistical significance are in bold.

$\mathrm{BMI}=$ body mass index; $\mathrm{EF}=$ ejection fraction; $I N R=$ international normalized ratio; $\mathrm{PTT}=$ partial thromboplastin time; Plt = platelet; $\mathrm{COPD}=$ chronic obstructive pulmonary disease; $\mathrm{CT}=$ cardiothoracic; $\mathrm{CPB}=$ cardiopulmonary bypass time.

thermia during DHCA and postoperative bleeding. Although some temperature variables correlated with postoperative bleeding in univariate analysis, none of these associations remained significant in multivariate analysis. These findings contrast with those of Parr et al., who demonstrated a link between postoperative bleeding and lowest temperature on CPB [22]. This discrepancy between studies may be explained by differences in patient cohorts, degrees of cooling on CPB, and variable use of DHCA; in addition, the extent of rewarming was not quantified in Parr et al.'s series.

Our operative strategy included active rewarming of the patient prior to coming off bypass. Such an approach is controversial, and the recent consensus statement regarding temperature management 
during CPB by Engelman et al. would appear to conflict with this technique [21]. However, these guidelines are not specific for DHCA, and each of the Class I recommendations is understandably limited to a Level of Evidence of $C$ (evidence from expert opinion, case studies, or standard of care). Hata et al. showed superior results in aortic cases requiring DHCA with active rewarming utilizing $40^{\circ} \mathrm{C}$ perfusion water bath [23]. We also relied on the temperature of the venous return to guide both our cooling and rewarming thresholds, in part due to the extreme variability in temperatures measured at surface locations on the body. As noted by Tian et al. [2] and Stone et al. [24], such variation among esophageal, pulmonary artery, rectal, and bladder temperatures may not reflect true cortical or core temperature. Our data support prior studies demonstrating an association between postoperative bleeding and re-operative surgery, duration of CPB, and COPD [21, 25].

Our study is limited by its size and retrospective design. All data were collected from a single center and therefore may not be widely generalizable. Because of the retrospective nature of this study, characterization of the full relationship between bleeding and the degree of rewarming was limited, as temperature at discontinuation from bypass did not vary widely in these patients. However, the relatively low transfusion rates suggests that the temperature management strategy employed in the face of DHCA might have merit. Each of the four different measures of postoperative bleeding has limitations. Furthermore, while our institution has guidelines for blood transfusion, the decision for transfusion was made on an individual basis. Stricter criteria would improve the reliability of transfusion as a surrogate for bleeding.

In conclusion, we found that intraoperative temperature measures were not predictive of postoperative bleeding. Therefore, the strategy for intraoperative temperature management should center on optimizing brain and end-organ protection for the duration required for surgery, and based on our experience, rewarming may reduce the risk of coagulopathy.

\section{Conflict of Interest}

The authors have no conflict of interest relevant to this publication.

\section{Comment on this Article or Ask a Question}

\section{References}

1. Rohrer MJ, Natale AM. Effect of hypothermia on the coagulation cascade. Crit Care Med. 1992;20:1402-1405. PMID: 1395660

2. Tian DH, Wan B, Bannon PG, Misfeld M, Lemaire SA, Kazui T, et al. A meta-analysis of deep hypothermic circulatory arrest versus moderate hypothermic circulatory arrest with selective antegrade cerebral perfusion. Ann Cardiothorac Surg. 2013;2:148-158. DOI: 10.3978/j. issn.2225-319X.2013.03.13

3. Stein LH, Elefteriades JA. Protecting the brain during aortic surgery: an enduring debate with unanswered questions. J Cardiothorac Vasc Anesth. 2010;24:316-321. DOI: $10.1053 /$ j.jvca.2009.05.016

4. Greeley WJ, Kern FH, Ungerleider RM, Boyd $\mathrm{JL}$, 3rd, Quill T, Smith LR, et al. The effect of hypothermic cardiopulmonary bypass and total circulatory arrest on cerebral metabolism in neonates, infants, and children. J Cardiothorac Vasc Anesth. 1991;101:783-794. PMID: 2023435

5. Staab DB, Sorensen VJ, Fath JJ, Raman SB, Horst HM, Obeid FN. Coagulation defects resulting from ambient temperature-induced hypothermia. J Trauma. 1994;36:634-638. PMID: 8189462

6. Thorsen K, Ringdal KG, Strand K, Soreide E, Hagemo J, Soreide K. Clinical and cellular effects of hypothermia, acidosis and coagulopathy in major injury. The Br J Surg. 2011;98:894907. DOI: 10.1002/bjs.7497

7. Michelson AD, MacGregor H, Barnard MR, Kestin AS, Rohrer MJ, Valeri CR. Reversible inhibition of human platelet activation by hypothermia in vivo and in vitro. Thromb Haemost. 1994;71:633-640. PMID: 7522354

8. Valeri $C R$, Feingold $H$, Cassidy $G$, Ragno G, Khuri S, Altschule MD. Hypothermiainduced reversible platelet dysfunction. Ann Surg. 1987;205:175-181. PMID: 3813688

9. Reed RL, 2nd, Bracey AW, Jr., Hudson JD, Miller TA, Fischer RP. Hypothermia and blood coagulation: dissociation between enzyme activity and clotting factor levels. Circ Shock. 1990;32:141-152. PMID: 2253317

10. Kheirabadi BS, Delgado AV, Dubick MA, Scherer MR, Fedyk CG, Holcomb JB, et al. In vitro effect of activated recombinant factor
VII ( $\mathrm{rFVIIa)} \mathrm{on} \mathrm{coagulation} \mathrm{properties} \mathrm{of} \mathrm{hu-}$ man blood at hypothermic temperatures. J Trauma. 2007;63:1079-1086. DOI: 10.1097/ TA.0b013e31815885f1

11. Ranucci M, Baryshnikova E, Castelvecchio S, Pelissero G, Surgical, Clinical Outcome Research G. Major bleeding, transfusions, and anemia: the deadly triad of cardiac surgery. Ann Thorac Surg. 2013;96:478-485. DOI: 10.1016/j.athoracsur.2013.03.015

12. Shore-Lesserson L, Reich DL, Silvay G, Griepp RB. Hemostasis in aortic and cardiothoracic surgery. J Card Surg. 1997;12:232-237. PMID: 9271751

13. Woodman RC, Harker LA. Bleeding complications associated with cardiopulmonary bypass. Blood. 1990;76:1680-1697. PMID: 2224118

14. Salis S, Mazzanti VV, Merli G, Salvi L, Tedesco CC, Veglia F, et al. Cardiopulmonary bypass duration is an independent predictor of morbidity and mortality after cardiac surgery. J Cardiothorac Vasc Anesth. 2008;22:814-822. DOI: $10.1053 /$ j.jvca.2008.08.004

15. Lopes CT, Brunori EH, Santos VB, Moorhead SA, Lopes JL, de Barros AL. Predictive factors 
for bleeding-related re-exploration after cardiac surgery: a prospective cohort study. Eur J Cardiovasc Nurs. 2016;15:e70-e77. DOI: $10.1177 / 1474515115583407$

16. Dixon B, Reid D, Collins $M$, Newcomb AE, Rosalion $\mathrm{A}$, Yap $\mathrm{CH}$, et al. The operating surgeon is an independent predictor of chest tube drainage following cardiac surgery. J Cardiothorac Vasc Anesth. 2014;28:242-246. DOI: 10.1053/j.jvca.2013.09.010

17. Smith D, Grossi EA, Balsam LB, Ursomanno $P$, Rabinovich A, Galloway AC, DeAnda A. The impact of a blood conservation program in complex aortic surgery. Aorta(Stamford) 2014;1:219-226. DOI: 10.12945/j. aorta.2013.13-035

18. Yaffee DW, DeAnda A, Ngai JY, Ursomanno PA, Rabinovich AE, Ward $A F$, et al. Blood conservation strategies can be applied safely to high-risk complex aortic surgery. J Cardiothorac Vasc Anesth. 2015;29:703-709. DOI: 10.1053/j. jvca.2014.10.022

19. Ferraris VA, Brown JR, Despotis GJ, Hammon JW, Reece TB, Saha SP, et al. Society of Thoracic Surgeons Blood Conservation Guideline Task Force, et al. 2011 update to the Society of Thoracic
Surgeons and the Society of Cardiovascular Anesthesiologists blood conservation clinical practice guidelines. Ann Thorac Surg. 2011;91:944-982. DOI: 10.1016/j. athoracsur.2010.11.078

20. Dyke C, Aronson S, Dietrich W, Hofmann $A$, Karkouti K, Levi $M$, et al. Universal definition of perioperative bleeding in adult cardiac surgery. J Thorac Cardiovasc Surg. 2014;147:1458-1463.e1. DOI: 10.1016/j. jtcvs.2013.10.070

21. Engelman R, Baker RA, Likosky DS, Grigore A, Dickinson TA, ShoreLesserson L, et al. The Society of Thoracic Surgeons, The Society of Cardiovascular Anesthesiologists, and The American Society of ExtraCorporeal Technology: clinical practice guidelines for cardiopulmonary bypass-temperature management during cardiopulmonary bypass. Ann Cardiothorac Surg. 2015;100:748-757. DOI: 10.1016/j.athoracsur.2015.03.126

22. Parr KG, Patel MA, Dekker R, Levin R, Glynn $\mathrm{R}$, Avorn J, et al. Multivariate predictors of blood product use in cardiac surgery. J Cardiothorac Vasc Anesth. 2003;17:176181. DOI: $10.1053 /$ jcan.2003.44

23. Hata M, Suzuki M, Sezai A, Niino T,
Unosawa S, Furukawa N, et al. Less invasive quick replacement for octogenarians with type A acute aortic dissection. J Thorac Cardiovasc Surg. 2008;136:489-493. DOI: 10.1016/j.jtcvs.2012.06.047

24. Stone JG, Young WL, Smith CR, Solomon RA, Wald A, Ostapkovich N, et al. Do standard monitoring sites reflect true brain temperature when profound hypothermia is rapidly induced and reversed? Anesthesiology. 1995;82:344-351. PMID: 7856892

25. Mistiaen W, Van Cauwelaert P, Muylaert P. Prediction of surgical outcome after aortic valve replacement. Acta Chirurgica Belgica. 2012;112:59-64. PMID: 22442911

Cite this article as: Stein $\mathrm{LH}$, Rubinfeld G, Balsam LB, Ursomanno P, DeAnda A. Too Cold to Clot? Does Intraoperative Hypothermia Contribute to Bleeding After Aortic Surgery? AORTA (Stamford). 2017;5(4):106-116. DOI: https://doi.org/10.12945/j.aorta.2017. 16.049 